\title{
Análisis de Beneficio de Incidencia: Implante coclear infantil en el Hospital Barros Luco Trudeau período 2003 a 2011
}

\author{
Benefit/Incidence Analysis: cochlear implants in children \\ at Barros Luco Trudeau Hospital, period 2003-2011
}

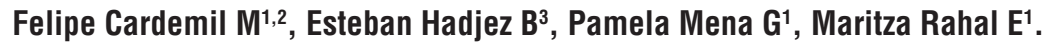

\begin{abstract}
RESUMEN
Introducción: La hipoacusia sensorioneural infantil es una causa frecuente de discapacidad en la infancia. Si bien la intervención ha demostrado ser costo efectiva, un alto porcentaje no accede al implante coclear por problemas de cobertura de los servicios de salud.

Objetivo: Conocer el uso y distribución del gasto público de salud en el programa piloto de implantes cocleares del Hospital Barros Luco Trudeau.

Material y método: Se realizó un Análisis de Beneficio de Incidencia, que permite conocer la distribución y el uso del gasto público en salud entre los distintos grupos socioeconómicos.

Resultados: Durante el período del estudio se realizaron 73 implantes cocleares en menores de 15 años, correspondiendo a 35 niñas y 38 hombres. El 56,1\% de los menores con implante coclear se ubicaban en el primer quintil de ingresos, el 27,5\% al segundo quintil, $15,1 \%$ en el tercer quintil, y un caso pertenecía al cuarto quintil, no registrándose ningún niño en el quinto quintil de ingresos según los datos de la encuesta CASEN 2009.

Discusión: Al evaluar el programa se deduce que en este caso específico los recursos públicos se encuentran focalizados hacia la población con menores ingresos económicos y con menor acceso a los servicios sanitarios.
\end{abstract}

Palabras clave: Hipoacusia infantil, implantes cocleares, gasto público en salud.

\begin{abstract}
Introduction: Childhood sensorineural hearing loss is a common cause of disability in children, with harmful consequences for individuals, family and society if it is not detected and treated early in life. While the intervention has proven to be cost effective, a high percentage does not access the cochlear implant coverage because of problems of health care.

\footnotetext{
Médico, Servicio de Otorrinolaringología, Hospital Barros Luco Trudeau - Universidad de Chile.

Programa de Doctorado en Salud Pública, Escuela de Salud Pública, Universidad de Chile.

3 Médico, Magíster en Salud, Comunidad y Desarrollo, LSE. Departamento de Salud Pública, Escuela de Medicina, Universidad de Valparaíso.
} 
Aim: To determine the use and distribution of public expenditure in health in the pilot program of cochlear implants of the Barros Luco Trudeau Hospital.

Material and method: We conducted a Benefit/Incidence Analysis, that is a model that evaluate the distribution and use of public expenditure on health between different socioeconomic groups.

Results: During the study period there were performed 73 cochlear implants in children under 15 years, corresponding to 35 girls and 38 men. The $56.1 \%$ of children with cochlear implants belonged to the lowest income quintile, $27.5 \%$ to the second quintile, $15.1 \%$ belonged to the third quintile, and one case belonged to the fourth quintile. There were no child of the fifth quintile of income according to the data of the 2009 CASEN survey.

Discussion: When evaluating the program, in this specific case we can establish that public resources are targeted to people with lower incomes and less access to health services.

Key words: Childhood Hearing Loss, Cochlear Implants, Public Health Expenditure.

\section{INTRODUCCIÓN}

La hipoacusia sensorioneural infantil es una de las causas de discapacidad más frecuentes en la infancia'. Su aparición en el período neonatal o en etapas posteriores del desarrollo del niño, permite clasificarlas en hipoacusias congénitas 0 adquiridas, y de acuerdo a su compromiso sensorial podemos reconocer condiciones leves, moderadas, severas y profundas $^{1,2}$. Si bien cualquier grado de compromiso auditivo en la infancia supone dificultades en la adquisición del lenguaje oral y en la interacción social, es en las dos últimas categorías de severidad donde se establecen y reproducen las mayores dificultades personales, familiares y sociales asociadas con la hipoacusia ${ }^{3,4}$.

En relación a lo anterior, la posibilidad de un diagnóstico precoz de hipoacusia sensorioneural a través de la pesquisa neonatal universal, y el inicio de tratamiento en las primeras etapas del desarrollo cognitivo mediante audífonos 0 implante coclear, permiten a los niños y niñas con hipoacusia mejorar significativamente el desarrollo del lenguaje oral y escrito, y su integración a la familia y la sociedad ${ }^{1,5,6}$. Si bien existen diversos estudios que reconocen la efectividad y la costo efectividad del implante coclear precoz en niños con hipoacusia sensorioneural severa y profunda ${ }^{7-10}$, un alto porcentaje de esta población infantil no accede a esta intervención, tanto por cobertura insuficiente en los servicios sanitarios ${ }^{11-13}$ como por factores económicos y étnicos de exclusión social ${ }^{13-16}$.
Por otra parte, existe evidencia acerca de la relación entre pobreza, marginalidad y mayor prevalencia de hipoacusia sensorioneural en población infantil2,17-19, y la reproducción de las inequidades sociales debido a los mayores índices de analfabetismo e inactividad laboral en las personas con discapacidad auditiva en nuestro país ${ }^{20}$.

\section{OBJETIVO}

El objetivo de este estudio fue analizar hacia qué grupos sociales se ha focalizado el uso de los recursos públicos del programa de implante coclear infantil en la población atendida en el Hospital Barros Luco Trudeau. Con esta información, se pretende evaluar en forma general la eficiencia de ese programa piloto de implante coclear, considerando la mayor prevalencia y al mismo tiempo las limitaciones en el acceso a los servicios de salud de las poblaciones más excluidas de la sociedad ${ }^{21}$.

\section{MATERIAL Y MÉTODO}

Se realizó un Análisis de Beneficio de Incidencia, modelo que permite conocer la distribución y el uso del gasto público en salud entre los distintos grupos socioeconómicos dentro de un país o región ${ }^{22,23}$. Para su construcción, se requiere de información sobre a) el gasto público del servicio estudiado, b) la utilización pública de dicho servicio y c) la 
caracterización socioeconómica de la población objetivo ${ }^{23}$.

De acuerdo a lo anterior, se consideraron todos los implantes cocleares en población infantil realizados en el Hospital Barros Luco Trudeau entre los años 2003 y 2011, la revisión de todas las fichas clínicas de los niños y niñas usuarios de este servicio, obteniéndose los datos sobre sexo, edad de implantación coclear, ingreso económico autónomo del hogar e ingreso per cápita. Por último se realizó la caracterización socioeconómica de toda la población de la región Metropolitana en quintiles de ingreso autónomo por hogar, a través de los datos de la encuesta CASEN 200920 (Tabla 1). Quintil es el nombre dado a los cinco grupos que clasifican a la población chilena por niveles de ingresos económicos: el quintil 1 corresponde al $20 \%$ de la población con menores ingresos, y el quintil 5 , al $20 \%$ de mayores ingresos. Para determinar el quintil per cápita se deben sumar todos los ingresos del hogar y dividirlos por la cantidad de integrantes de la familia.

Para el análisis de la información, se tabularon los datos y se incorporaron los datos de ingreso per cápita de los menores con implante coclear en la caracterización por quintiles de ingreso autónomo. Posteriormente, se obtuvo el número total de implantes cocleares por cada quintil de ingreso autónomo per cápita y se graficó la relación entre estas dos variables. Para el objetivo de este trabajo, que era conocer la distribución y el uso del programa de implante coclear infantil, se omitió la estimación del precio de esta intervención, y se mantuvo la cantidad total de implantes como expresión del uso por parte de la población en estudio.

\section{RESULTADOS}

Durante el período del estudio, en el Hospital Barros Luco Trudeau se realizaron 73 implantes cocleares en menores de 15 años, correspondiendo a 35 niñas $(47,9 \%)$ y 38 niños $(52,1 \%)$, con una edad promedio de colocación del implante de 4 años y un mes, una mediana de 3 años y 6 meses, y un rango de 10 meses a 13 años. El 80,8\% de los menores (59 casos) recibió el implante coclear antes de los 6 años, y el 34,2\% (25 casos) antes de los 3 años.

El promedio de ingresos autónomos por hogar fue de $\$ 351.207$, con una mediana de $\$ 251.860$, un ingreso mínimo de $\$ 48.000$ y un ingreso máximo de $\$ 970.000$. El ingreso per cápita promedio fue de $\$ 82.583$, con una mediana de $\$ 65.934$, un ingreso per cápita mínimo de $\$ 8.143$ y un máximo de $\$ 233.333$. De acuerdo a estas últimas cifras, el $56,1 \%$ de los menores con implante coclear (41 casos) se ubicaban en el primer quintil de ingresos, el $27,5 \%$ (20 casos) correspondieron al segundo quintil de ingresos, el 15,1\% (11 casos) se ubicaban en el tercer quintil de ingresos, y un caso pertenecía al cuarto quintil de ingresos, no registrándose ningún niño o niña en el quinto quintil de ingresos autónomos per cápita de acuerdo a los resultados de la encuesta CASEN 2009. En la Figura 1 la línea discontinua representa una situación

Tabla 1. Quintiles de promedio de ingreso autónomo por hogar y per cápita en sectores urbanos de la Región Metropolitana (ingreso en pesos a noviembre del 2009)

\begin{tabular}{|l|c|c|}
\hline Quintil de Ingreso & Promedio ingreso autónomo hogar & Promedio ingreso per cápita del hogar \\
\hline & & \\
\hline I & $\$ 184.920$ & $\$ 43.926$ \\
\hline II & $\$ 382.597$ & $\$ 99.722$ \\
\hline III & $\$ 596.623$ & $\$ 161.734$ \\
\hline IV & $\$ 937.731$ & $\$ 280.671$ \\
\hline V & $\$ 2.875 .997$ & $\$ 1.104 .034$ \\
\hline
\end{tabular}

Tomado de: MIDEPLAN, Ministerio de Planificación. Encuesta de Caracterización Socioeconómica (CASEN) 2009. Santiago, Chile. 


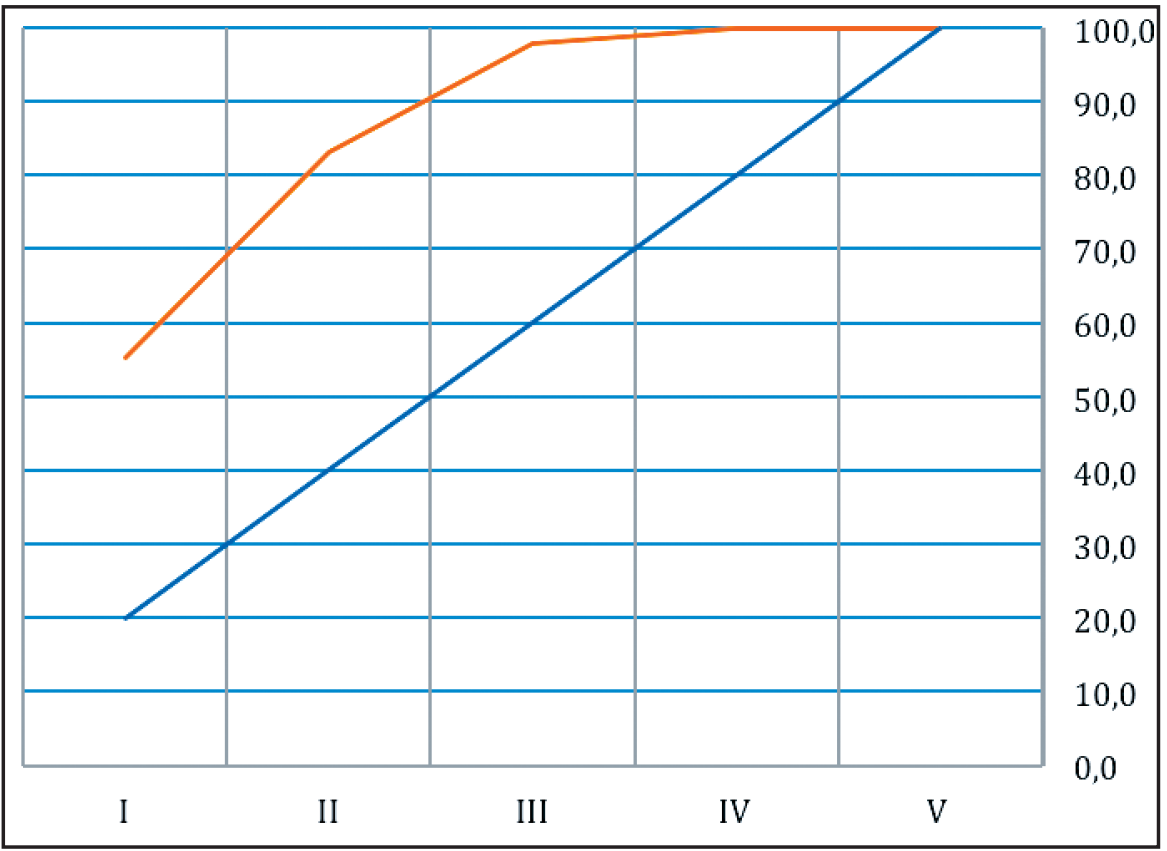

Figura 1. Distribución del número de implantes cocleares (\%) en relación a los quintiles de ingreso autónomo per cápita de los hogares de la región Metropolitana (línea continua) entre los años 2003 y 2011.

de relación directamente proporcional, en la cual cada uno de los quintiles posee el mismo número de beneficiarios, correspondiendo al $20 \%$ en cada quintil. La línea continua muestra el porcentaje de beneficiados por quintil de ingreso. El promedio de integrantes por hogar fue de 4,4 personas, una mediana de 4, con un mínimo de 3 integrantes por hogar y un máximo de 7.

\section{DISCUSIÓN}

En relación a la distribución de la población infantil que recibe un implante coclear en el Hospital Barros Luco Trudeau, y su caracterización en quintiles de ingresos autónomos per cápita, se puede mencionar que el gasto público de este programa de implante coclear se ha focalizado hacia la población de menores ingresos económicos, donde existiría una mayor prevalencia de hipoacusia sensorioneural infantil, a diferencia de lo que se reporta en un trabajo de Stern RE y cols, el que usando datos de 1997 del Health Care and Utilization Project/Kids' Inpatient Database ${ }^{14}$ de Estados Unidos, donde se aprecia una disparidad en el acceso al beneficio, siendo niños blancos o asiáticos con padres con ingresos superiores al promedio quienes recibieron implantes a mayores tasas de las que cabría esperar sobre la base de la prevalencia de su pérdida auditiva neurosensorial. Los datos de ese trabajo no permiten a los autores determinar las causas de estas diferencias. Geers y Brenner ${ }^{15}$ presentaron resultados de una encuesta a familias de Estados Unidos y Canadá que buscaron un implante coclear para su hijo, encontrando que la mayoría era de origen étnico blanco y tenían más educación y mayores ingresos que la población general.

En el presente caso, son niños pertenecientes a los primeros quintiles de ingreso aquellos que tienen un mayor acceso al programa de implante coclear, por lo que corresponde a una política pública de salud progresiva y redistributiva (Figura 1).

De acuerdo a la evidencia disponible, es relevante el número de niños y niñas de esta serie que son intervenidos durante el período crítico de desarrollo del lenguaje, lo que permitiría plantear que se podrían esperar mejores resultados cognitivos, familiares y sociales a mediano y largo plazo $0^{1,3,5,6,15}$.

Es necesario señalar algunas limitaciones del presente estudio. En primer lugar, el gasto público 
en salud se ha relacionado únicamente con la presencia de implante coclear en la población infantil, y no se han considerado los gastos asociados a técnicas diagnósticas, a eventuales complicaciones del implante coclear, y al seguimiento multidisciplinario de la hipoacusia sensorioneural infantil. De hecho, está descrito que en condiciones de adecuada protección financiera en salud, son los niños pertenecientes a familias con menor nivel socioeconómico los que presentan una mayor tasa de complicaciones secundarias al implante, y menor adherencia al seguimiento del equipo de salud $^{16}$. En segundo lugar, la mayor utilización del programa de implante coclear infantil por parte de los sectores económicos más vulnerables, puede estar relacionada únicamente con una mayor prevalencia de la hipoacusia sensorioneural infantil en esta población, sin reflejar necesariamente un mejor acceso a los servicios sanitarios. Por estos motivos, es aún más importante realizar estos monitoreos y seguir analizando los datos existentes que permitan evaluar el impacto de los programas de salud.

Estos datos permiten plantear que, en términos de un programa piloto de salud, es necesaria la adecuada focalización de los recursos en salud, y más específicamente las intervenciones diagnósticas y terapéuticas en hipoacusia hacia los niños y niñas más vulnerables de la sociedad ${ }^{24}$, para así atenuar los perjudiciales efectos económicos y sociales de mediano y largo plazo asociados a la hipoacusia sensorioneural infantil, y a su vez disminuir las brechas sanitarias existentes en nuestra sociedad. Luego, al implementar programas universales, adquieren mayor relevancia aspectos técnicos como la cobertura, seguimiento, y tasa de contrarreferencia, además que por ser programas de carácter universal, la focalización de la política pierde relevancia.

\section{CONCLUSIÓN}

A través del Análisis de Beneficio de Incidencia en el programa de implante coclear infantil del Hospital Barros Luco Trudeau, entre los años 2003 y 2011, se puede plantear que se ha enfocado hacia los sectores con menores ingresos económicos, con eventual mayor prevalencia de hipoacusia sensorioneural infantil, y con menor acceso a los servicios sanitarios en la población beneficiaria del Servicio de Salud Metropolitano Sur. El Análisis de Beneficio de Incidencia es una herramienta útil para conocer la distribución y el uso real de los recursos públicos por parte de los distintos sectores económicos de la población, permitiendo la evaluación de los programas de salud pública y la reorientación de éstos hacia los sectores más vulnerables de la sociedad.

\section{BIBLIOGRAFÍA}

1. Smith RJ, Bale JF, JR., White KR. Sensorineural hearing loss in children. Lancet 2005; 365: 87990.

2. Mathers C, Smith A, Concha M. Global burden of hearing loss in the year 2000. In: Paper GW, ed. Geneva: World Health Organization; 2003.

3. Monsalve A, Núñez F. La importancia del diagnóstico e intervención temprana para el desarrollo de los niños sordos. Los programas de detección precoz de la hipoacusia. Intervención Psicosocial 2006; 15: 7-28.

4. Hualte F, Brannstrom J, Gerdtham UG. Societal costs of hearing disorders: a systematic and critical review of literature. International Journal of Audiology 2012; 51: 655-62.

5. Fortunato-Tavares T, Befi-Lopes D, Bento RF, AndRADE CR. Children with cochlear implants: communication skills and quality of life. Brazilian Journal of Otorhinolaryngology 2012; 78: 15-25.

6. Anmyr L, Olsson M, Larson K, Freidd A. Children with hearing impairment--living with cochlear implants or hearing aids. International Journal of Pediatric Otorhinolaryngology 2011; 75: 844-9.

7. Cheng AK, Rubin HR, Powe NR, Mellon NK, Francis HW, Niparko JK. Cost-utility analysis of the cochlear implant in children. JAMA: the Journal of the American Medical Association 2000; 284: 850-6.

8. Colgan S, Gold L, Wirth K, et al. The costeffectiveness of universal newborn screening for bilateral permanent congenital hearing impairment: systematic review. Academic Pediatrics 2012; 12: 171-80.

9. Schroeder L, Petrou S, Kennedy C, et al. The economic costs of congenital bilateral permanent 
childhood hearing impairment. Pediatrics 2006; 117: 1101-12.

10 Bond M, Mealing S, Anderson R, et al. The effectiveness and cost-effectiveness of cochlear implants for severe to profound deafness in children and adults: a systematic review and economic model. Health Technology Assessment 2009; 13: 1-330.

11 Garber S, Ridgely MS, Bradley M, Chin KW. Payment under public and private insurance and access to cochlear implants. Archives of Otolaryngology--Head \& Neck Surgery 2002; 128: $1145-52$.

12. McManus MA, Levtov R, White KR, Forsman I, Foust T, THompson M. Medicaid reimbursement of hearing services for infants and young children. Pediatrics 2010; 126 Suppl 1: S34-42.

13. Bradham T, Jones J. Cochlear implant candidacy in the United States: prevalence in children 12 months to 6 years of age. International Journal of Pediatric Otorhinolaryngology 2008; 72 : 1023-8.

14. Stern RE, Yueh B, Lewis C, Norton S, Sie KC. Recent epidemiology of pediatric cochlear implantation in the United States: disparity among children of different ethnicity and socioeconomic status. The Laryngoscope 2005; 115: 125-31.

15. Geers A, Brenner C. Background and educational characteristics of prelingually deaf children implanted by five years of age. Ear and Hearing 2003; 24: 2S-14S.

16. Chang DT, Ko AB, Murray GS, Arnold JE, Megerian CA. Lack of financial barriers to pediatric cochlear implantation: impact of socioeconomic status on access and outcomes. Archives of Otolaryngology--Head \& Neck Surgery 2010; 136: 648-57.
17. Czechowicz JA, Messner ah, Alarcon-Matutti E, ET AL. Hearing impairment and poverty: the epidemiology of ear disease in Peruvian schoolchildren. Otolaryngology--Head and Neck Surgery: Official Journal of American Academy of Otolaryngology-Head and Neck Surgery 2010; 142: 272-7.

18. Menra S, Eavey RD, Keamy DG, Jr. The epidemiology of hearing impairment in the United States: newborns, children, and adolescents. Otolaryngology--Head and Neck Surgery: Official Journal of American Academy of OtolaryngologyHead and Neck Surgery 2009; 140: 461-72.

19. Olusanya BO, Newton VE. Global burden of childhood hearing impairment and disease control priorities for developing countries. Lancet 2007; 369: 1314-7.

20. MIDEPLAN MdP. Encuesta de caracterización socio económica. In. Santiago, Chile; 2009.

21. Barr NA. The economics of the welfare state. 4 th ed. Oxford: Oxford University Press; 2004.

22. Castro-Leal F, Dayton J, Demery L, Mehra K. Public spending on health care in Africa: do the poor benefit? Bulletin of the World Health Organization 2000; 78: 66-74.

23. Davoodi hR, Tiongson E, Asawanuchit SS, International Monetary Fund. Fiscal Affairs Dept., International Monetary Fund. Middle Eastern Department. How useful are benefit incidence analyses of public education and health spending? Washington, D.C.: International Monetary Fund, Fiscal Affairs Department and Middle Eastern Department; 2003.

24. GWATKIN DR. Health inequalities and the health of the poor: what do we know? What can we do? Bulletin of the World Health Organization 2000; 78: 3-18. 